\title{
Supercritical hydrothermal synthesis of silver nanoparticles, composites, and their characterizations
}

\author{
Narandalai Byamba-Ochir, Nemekhbayar Davaadorj, Battseveen Buyankhishig \\ and Enkhtuul Surenjav*
}

Institute of Chemistry and Chemical Technology, Mongolian Academy of Sciences, Ulaanbaatar 13330, Mongolia

*Corresponding author: enkhtuul@mas.ac.mn; ORCID iD: 0000-0002-2357-5339

Received: 08 October 2021; revised: 10 December 2021; accepted: 11 December 2021

\begin{abstract}
Silver nanoparticles (AgNPs) and silver nanoparticles doped activated carbon (AC-Ag) composite materials were synthesized by hydrothermal processes in supercritical water conditions ( $29 \mathrm{MPa}$ and $400{ }^{\circ} \mathrm{C}$ ) using batch reactor. We studied the influence of the precursor solution concentration, reaction temperature under the hydrothermal conditions, and synthesis time on the properties of synthesized materials. The properties of plain AgNPs and AC-Ag composite materials synthesized in supercritical water, including crystallinity, particle size, and molecular interactions between $A C$ and $A g$ were investigated, comprehensively. Compared to the plain AgNPs, the activated carbon-supported Ag nanocomposite was synthesized faster due to the active functional groups of activated carbon. Furthermore, the FTIR results reveal that the silver nanoparticles are attached to the activated carbon surface in the presence of oxygen bonded carbonyl and carboxyl groups. The nano-sized metal silver particles were observed on the AC surface when analyzed by TEM and $\mathrm{XRD}$. All results imply that the supercritical water condition allows the formation of silver particles less than $100 \mathrm{~nm}$ either in the form of plain particles or deposited on the activated carbon surface using the silver acetate precursor solution. This environmentally benign supercritical hydrothermal process can replace the conventional method and become a novel synthesis method for preparing various new materials.
\end{abstract}

Keywords: supercritical hydrothermal synthesis, activated carbon, silver nanoparticles, characterization

\section{INTRODUCTION}

The hydrothermal reactions for the formation of particles are solvothermal reactions where water is used as a solvent [1]. Due to the drastic change of water properties near the critical point, the solubility of metal salt, which is used as a precursor solution for hydrothermal synthesis, is greatly enhanced around the critical point of water; thus, reaction equilibrium and kinetics will also change. By employing those unique properties of supercritical conditions, the supercritical hydrothermal synthesis was studied successfully for metal and metal oxide nanoparticles synthesis [2, 3]. The hydrothermal technique is ideal for the processing of fine particles having high purity, controlled stoichiometry, narrow particle size distribution, uniformity, high crystallinity, high reactivity with ease of sintering, and so on, but it has a controlled micro-sized structure [4], and it takes long reaction time [5]. Therefore, after obtaining micro-sized particles, it's necessary to conduct thermal treatment and need additional chemicals such as organic solvents, reducing agents, or dispersants to get nano-sized materials [6].

The supercritical water (SCW) provides an excellent reaction medium for hydrothermal processing of nanoparticles since it allows varying the reaction rate and equilibrium by shifting the dielectric constant and solvent density for pressure and temperature, thus resulting in higher reaction rates and smaller particles. The reaction products are to be stable in SCW, leading to fine particle formation [2]. Hydrothermal process conditions of temperature and pressure can be varied in sub- and super-critical water conditions for the synthesis of metal oxide nanoparticles. The conventional hydrothermal reaction rate is enhanced more than $10^{3}$ times under hydrothermal synthesis in supercritical water [7]. Because the SCW has a low viscosity and high diffusivity facilitates the penetration of water into the complex structure of the metallic salt, 
whilst its low dielectric constant $(<10)$ enhances the solubility of organic compounds constant as well as products with high crystallinity [8].

SCW condition also allows the nanoparticles to deposit on a support surface and inside the pores in well-dispersed conditions [9]. Furthermore, carbonsupported metal oxides are of interest in an adsorption technique because porous activated carbon can be incorporated with metal oxides by various functional groups on the own surface, and it has a large specific surface area and pore distributions that are required for the removal of pollutants [10-12]. Due to these advantages, the use of activated carbon, often as a composite material with metal oxides, in wastewater treatment is increasing dramatically [13]. Activated carbon composite materials are excellent adsorbents, catalysts, sensors, medical, cosmetics, and water treatments. Among the activated carbon-supported composite materials, silver nanoparticles in the nanometer size range show a very high antibacterial activity compared with bulk ones for medical and drinking water treatment.

We aim to study the synthesis of new materials, including silver nanoparticles and silver nanoparticle-doped activated carbon composites, using a simple laboratory apparatus that performs supercritical hydrothermal synthesis. The formation of silver particles and silver particles doped activated carbon materials and their characterization, including particle size, crystallinity, surface properties, were studied.

\section{EXPERIMENTAL}

Materials: Silver acetate $\left(\mathrm{CH}_{3} \mathrm{COOAg}, 99.5 \%\right.$ purity) of analytical grade was purchased from Shanghai Macklin Biochemical Co.Ltd. Activated carbons (ACs) used in this research were prepared from Mongolian natural semi-anthracite, which has been chemically treated with $\mathrm{KOH}$. The preparation procedure of ACs was described in our previous publication [14].

Apparatus: The synthesis reaction was performed in a pressure-resistant tube reactor with an inner volume of $5.0 \mathrm{~mL}$ (Model INC 315) equipped with an automatic stirrer and a temperature-controlled electric furnace (Nabertherm) and a cooling water bath for quenching the reactor to terminate the reaction.

Synthesis of the silver nanoparticles (AgNPs): The precursor solution with a concentration of 0.01 $\mathrm{M}, 0.02 \mathrm{M}, 0.03 \mathrm{M}$, and $0.05 \mathrm{M}$ was prepared. The 3.0 $\mathrm{mL}$ of precursor solution with various concentrations was loaded in the batch reactor. The formation of silver nanoparticles from precursor solution was carried out in a batch reactor inserted in an electric furnace at a selected temperature in the range of 250 ${ }^{\circ} \mathrm{C}$ (hydrothermally) to $450{ }^{\circ} \mathrm{C}$ (at the supercritical temperature of water). The heating rate was optimized as $12.67^{\circ} \mathrm{C} / \mathrm{min}$ and the reaction time of synthesis was $5,10,15$, and $30 \mathrm{~min}$. After the reaction time, the batch reactor with the sample was quenched in the cooling water bath. The synthesis product was washed by distilled water and dried at $110^{\circ} \mathrm{C}$ in a vacuum oven overnight.

Hydrothermal synthesis of silver nanoparticles at supercritical water conditions was obtained in the following order: first, the hydrolysis of silver acetate salt occurred, then followed by the dehydration process, and finally, the transformation of silver oxide to metal silver as described in the literature [15].

Synthesis of the silver nanoparticles doped activated carbon nanocomposite (AC-Ag): Briefly, $3 \mathrm{ml}$ of precursor solution $\left(\mathrm{CH}_{3} \mathrm{COOAg}\right)$ of appropriate concentrations and $0.06 \mathrm{~g}$ of activated carbon powders were mixed with a ratio of 12:1. The solution was then transferred to a batch reactor and placed in the preheated electric furnace at the temperature range of $250{ }^{\circ} \mathrm{C}-450{ }^{\circ} \mathrm{C}$, where the reaction times varied between 5 to 30 mins. The synthesis condition of the silver particles on the surface of AC particles is the same as the previous experiment of synthesis of plain AgNPs, and the resulting silver particles are loaded to the activated carbon surface via the interaction of the oxygen in the hydroxyl and carboxyl groups.

Characterization of the synthesized products: The crystallite sizes of prepared silver particles AgNPs and AgNPs deposited on the activated carbon surface was calculated from the XRD data where X-ray powder diffraction was carried out on the Maxima_X XRD-7000 with monochromated $\mathrm{Cu}$ Ka radiation $(\lambda=1.5418 \AA)$. The mean size of crystallite (D) was evaluated from the following Scherrer equation [16]:

$$
D=\frac{k \lambda}{F W H M \cos \theta}
$$

where $k$ is the Scherrer constant set to $0.89, \lambda$ is the wavelength of the X-ray radiation, FWHM is the full width at half maximum for the diffraction peaks, and $\theta$ is the diffraction angle.

The morphologies of the obtained particles were studied by using scanning electron microscopy (Topcon SM-300 electron microscope operated at $10 \mathrm{kV}$ ) and high-resolution transmission electron microscopy (2000FXII; JEOL TEM, Japan). The surface chemical characterization of the AC-Ag samples and the primary activated carbon sample was done by the Raman spectrometer (Horiba LabRam HR Evolution Raman microscope) and the XPS (Multilab 2000 / Thermo electron Corp., England) spectrometer.

\section{RESULTS AND DISCUSSION}

\section{Synthesizing AgNPs and AC-Ag nanocomposites} under the supercritical hydrothermal condition: The representative TEM images of synthesized AgNPs and the AC-Ag nanocomposite, which were prepared at $400{ }^{\circ} \mathrm{C}$ temperature and reaction time of 30 mins when using $0.01 \mathrm{M} \mathrm{CH}_{3} \mathrm{COOAg}$ are shown in Fig. 1 . 

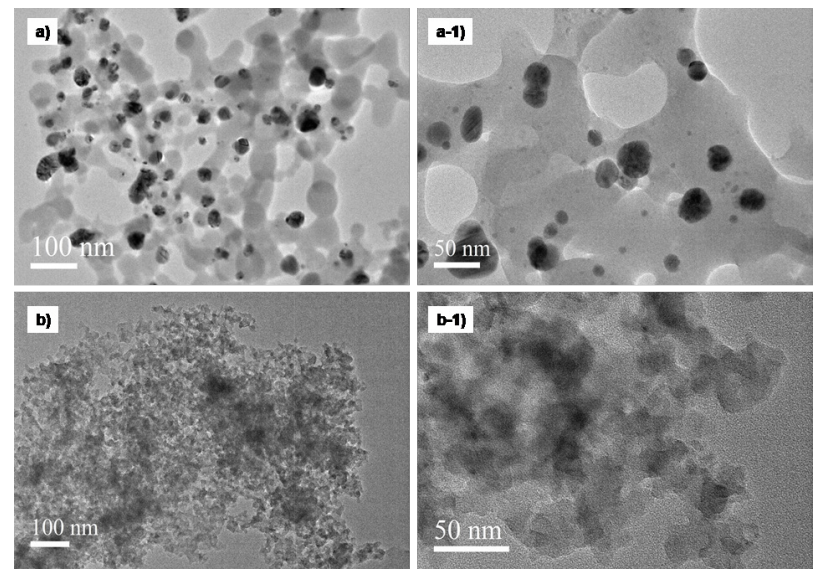

Fig. 1. TEM image of the synthesized AgNPs (a; a-1) and AC-Ag nanocomposite (b; b-1). The synthesis condition is as follows: the concentration of precursor solution is $0.01 \mathrm{M}$, the reaction temperature is 400 ${ }^{\circ} \mathrm{C}$ and the reaction time is $30 \mathrm{~min}$

As represented in Fig. 1(a. and a-1.), the silver particles (AgNPs) with a single crystalline structure with an average particle size of 25 - $50 \mathrm{~nm}$ were synthesized from $0.01 \mathrm{M}$ silver acetate solution at $400{ }^{\circ} \mathrm{C}$ for 30 minutes. In addition, there was no agglomeration of the silver particles observed under these optimal conditions. In the case of composite materials synthesis, the silver particles were well deposited on the surface of ACs where the black dots represent the AgNPs, while the grey parts trace the dispersed solvent cf. Fig. 1 (b and $\mathrm{b}-1)$. Due to the trace of the dispersed solvent, the particles were not seen clearly.

X-ray diffractometer's analyses of AgNPs, which were synthesized at different temperatures (from $250{ }^{\circ} \mathrm{C}$ to $450{ }^{\circ} \mathrm{C}$ ) were performed to study the temperature influence of hydrothermal conditions [15]. The reaction time was $30 \mathrm{~min}$, and the heating rate was $12.67^{\circ} \mathrm{C} /$ min during the synthesis. XRD results indicated no silver peak at $250{ }^{\circ} \mathrm{C}$, but silver and silver acetate peaks illustrated at $300{ }^{\circ} \mathrm{C}$ and $350{ }^{\circ} \mathrm{C}$, due to the decomposition of acetate under hydrothermal conditions. However, the experiments conducted at $400{ }^{\circ} \mathrm{C}$ and $450{ }^{\circ} \mathrm{C}$ revealed no peak of silver acetate, suggesting that silver nanoparticles could be synthesized only in SCW media. In other words, at super-critical point temperatures above $374{ }^{\circ} \mathrm{C}$ of the water phase, the decomposition of acetate takes place in a relatively short time, allowing the formation of silver particles.

Therefore, the optimum temperature of hydrothermal synthesis was $400{ }^{\circ} \mathrm{C}$ both for plain silver particles and the AC-Ag composite materials for further experiments. Effect of the precursor solution concentration on the crystallite size of AgNPs: In this experiment, the concentration of the precursor solution of silver acetate salt was tested as $0.01 \mathrm{M}, 0.02 \mathrm{M}, 0.03 \mathrm{M}$, and $0.05 \mathrm{M}$, and the reaction temperature was kept at $400{ }^{\circ} \mathrm{C}$ for 30 min reaction time.

Using X-ray diffractometer peaks of the synthesized silver particles with different concentrations, the crystallite size of silver particles was calculated by Scherrer's equation (Eq.1). The resulting average crystallite size was summarized in Table 1.

In Table 1, the average crystallite size of the synthesized silver nanoparticles gradually increased from $56.0 \mathrm{~nm}$ to $67.7 \mathrm{~nm}$ with an increasing concentration of the silver salt solution. In the case of silver synthesized on the surface of activated carbons nanocomposite inversely, the average crystallite size was decreased as the concentration of the precursor solution increased, which may have been influenced by the functional groups of the surface of the activated carbon.

Effect of the reaction time on the crystallization of AgNPs: The effect of the reaction time on the formation of AgNPs was studied at 15 and 30 mins of reaction time when precursor solution concentration was 0.01 $\mathrm{M}$. The comparative X-ray diffractometer's pattern of the obtained samples is shown in Fig. 2.

As illustrated clearly on the XRD pattern (Fig. 2) the peaks of silver acetate crystals were found in the range of 2 thetas $21-36^{\circ}$ for a shorter reaction time of 15 minutes for plain AgNPs sample. This indicates that the silver acetate is not completely decomposed within 15 min of reaction time. However, when reaction time is increased further to 30 minutes, the decomposition of silver acetate is completed, and single silver crystal peaks are detected, so the reaction time of 30 minutes is the appropriate time to synthesize pure silver particles. In the case of AC-Ag nanocomposite, 15 min of reaction

Table 1. The crystallite size of AgNPs and AC-Ag nanocomposites synthesized at $400{ }^{\circ} \mathrm{C}$ for 30 min with various precursor solution concentration

\begin{tabular}{llllll}
\hline No & Sample ID & $\begin{array}{l}\text { Temp., } \\
{ }^{\circ} \mathrm{C}\end{array}$ & $\begin{array}{l}\mathrm{C}_{\mathrm{CH}_{3} \mathrm{COOAg}_{\mathrm{g}},} \\
\mathrm{M}\end{array}$ & $\begin{array}{l}\text { Reaction } \\
\text { time, } \\
\mathrm{min}\end{array}$ & $\begin{array}{l}\text { Average diameter, } \\
\mathrm{nm}\end{array}$ \\
\hline 1 & Ag-0.01-400-30 & 400 & 0.01 & 30 & 56.0 \\
2 & Ag-0.02-400-30 & 400 & 0.02 & 30 & 55.4 \\
3 & Ag-0.03-400-30 & 400 & 0.03 & 30 & 60.7 \\
4 & Ag-0.05-400-30 & 400 & 0.05 & 30 & 67.7 \\
5 & AC-Ag-0.01-400-30 & 400 & 0.01 & 30 & 66.8 \\
6 & AC-Ag-0.02-400-30 & 400 & 0.02 & 30 & 55.5 \\
7 & AC-Ag-0.03-400-30 & 400 & 0.03 & 30 & 39.0 \\
\hline 8 & AC-Ag-0.05-400-30 & 400 & 0.05 & 30 & 27.0 \\
\hline
\end{tabular}




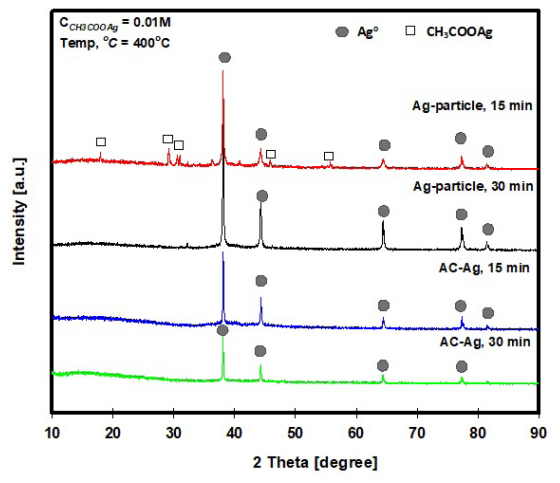

Fig. 2. XRD patterns of the AgNPs and AC-Ag at the reaction time of 15 and 30 min

time is enough for the complete decomposition of silver acetate, as shown in Fig. 2. AC-Ag composites show obvious peaks at $2 \theta=38.1^{\circ}, 44.3^{\circ}, 64.4^{\circ}, 77.4^{\circ}$, and $81.5^{\circ}$, which were caused by the face-centred cubic crystalline silver, corresponding to the crystal faces of (111), (200), (220), (311) and (222) revealing that the silver nanoparticles were well dispersed on the surface of ACs. This is in agreement with Kumar et al results on characterizations of silver nanowires synthesized by microwave-assisted technology [17]. The shorter time needed for AC-Ag nanocomposite is due to the functional group of activated carbon facilitating the faster deposition of a silver particle on the surface of activated carbon.

Effect of the surface properties of ACs on the crystallization of AgNPs: In the preparation of ACAg nanocomposite, we used activated carbon derived from Mongolian anthracite. The BET surface area of AC is $1227 \mathrm{~m}^{2} / \mathrm{g}$, the micropore volume is $0.61 \mathrm{~cm}^{3} / \mathrm{g}$, the micropore size is $0.53 \mathrm{~nm}$, and the mesopore size is $2.7 \mathrm{~nm}[14]$.

The results of the XPS analysis of the C1s and O1s region of the ACs samples indicating the carbon and oxygen concentration, are shown in Table 2.

According to the XPS results, the surface of the activated carbon sample had the highest graphic $(\mathrm{C}=\mathrm{C}$, $\mathrm{sp}^{2}$ and $\mathrm{C}-\mathrm{C}, \mathrm{sp}^{3}$ ) content of $89.43 \%$ of the atomic content of carbon $(\mathrm{C} 1 \mathrm{~s})$ from the functional groups.
In terms of the atomic content of oxygen $(\mathrm{O} 1 \mathrm{~s})$, the carbonyl functional groups $(>\mathrm{C}=\mathrm{O})$ are dominated by oxygen (47.96\%, atom), the oxygen content of quinine, and secondary carbonyl bonds are $23.25 \%$. The oxygen content of atoms, esters, and anhydrides is $18.79 \%$, and carboxylic oxygen is $5.68 \%$.

From the XPS results, the chemical properties of the activated carbon surface imply that containing oxygen functional groups (carbonyl, carboxyl, quinine groups), so silver particles formed under the supercritical hydrothermal condition can interact with these active groups by chemical bonding.

\section{Characterization of silver loaded AC composite} (AC-Ag): The morphology of the synthesized AgNPs on the surface of ACs has been compared with primary $A C$ and $A C$ treated in SCW condition cf. Fig. 3.

From Fig. 3( $a$ and $b$ ) no significant differences were observed in the morphology of primary activated carbon and activated carbon treated under supercritical water conditions. An enlarged SEM image in Fig. 3(c) shows that silver nanoparticles with a size of approximately 80 $\mathrm{nm}$ are doped with a chain-like interconnection on the surface layer of activated carbon. For better illustration, we have used the highly magnified image for the AC-Ag composite.

The elemental mapping for $\mathrm{Ag}, \mathrm{Si}$, and $\mathrm{Al}$ was performed by the EDX analysis (not shown) and found a good distribution of all the elements in the nanocomposite with their approximate stoichiometry $(\mathrm{Ag}-71.1 \%, \mathrm{Si}-$ $15.0 \%$, and $\mathrm{Al}-13.9 \%$ ).

Raman spectra analysis: The Raman spectra of primary activated carbons (Raw AC), activated carbons treated in SCW, and the synthesized silver particles on the activated carbon surface (AC-Ag-0.01-400-30) from $0.01 \mathrm{M}$ silver acetate solution are shown in Fig. 4 and the interpretation is shown in Table 3. The Raman spectrometer (Horiba Lab Ram HR Evolution Raman microscope) analysis was conducted at shift region between $500 \mathrm{~cm}^{-1}$ and $2300 \mathrm{~cm}^{-1}$.

As can be seen from the Raman spectra (Fig. 4), the three samples analyzed have two main bands ( $G$ and $D)$, and the peak ("G-band") on the 1589.2-1590.5 $\mathrm{cm}^{-1}$ shift represents the ordered graphitic structure

Table 2. High-resolution C1s and O1s XPS spectral peaks of the primary activated carbons

\begin{tabular}{|c|c|c|c|c|c|}
\hline Orbital & Binding Energy, eV & Functional groups & Bond & At. $\%$, conc. & $\mathrm{Co} / \mathrm{Cg}$ \\
\hline \multirow{4}{*}{ C1s } & $284.6-285.9$ & Graphitic & $\mathrm{C}=\mathrm{C} ; \mathrm{C}-\mathrm{C}$ & 89.43 & \multirow{9}{*}{0.31} \\
\hline & $286.0-286.9$ & Phenolic, alcoholic, etheric & $\mathrm{C}-\mathrm{O}-\mathrm{C}$ & 5.73 & \\
\hline & $283.19-283.87$ & Metal carbide & $\mathrm{MeC}$ & 2.25 & \\
\hline & $288.1-289.1$ & Carboxyl/ester & $\mathrm{COOH}$ or $\mathrm{HO}-\mathrm{C}=\mathrm{O}$ & 2.59 & \\
\hline \multirow{5}{*}{ 01s } & $532.2-532.9$ & Carbonyl oxygen & $>\mathrm{C}=\mathrm{O}$ & 47.96 & \\
\hline & $533.1-533.9$ & Ether type oxygen in esters, anhydrides & $\begin{array}{l}\mathrm{C}-\mathrm{O} \\
\mathrm{O}=\mathrm{C}-\mathrm{OH}\end{array}$ & 18.79 & \\
\hline & $534.3-534.9$ & Carboxyl & $\mathrm{O}=\mathrm{C}-\mathrm{OH}$ & 5.68 & \\
\hline & $531.26-531.82$ & $\begin{array}{l}\text { Oxygen doubly bonded to aromatic C, } \\
\text { (carbonyl oxygen of quinines) }\end{array}$ & $-\mathrm{C}(=\mathrm{O})-$ & 23.25 & \\
\hline & 529.98 & Metallic oxide & $\mathrm{MeO}$ & 4.32 & \\
\hline
\end{tabular}



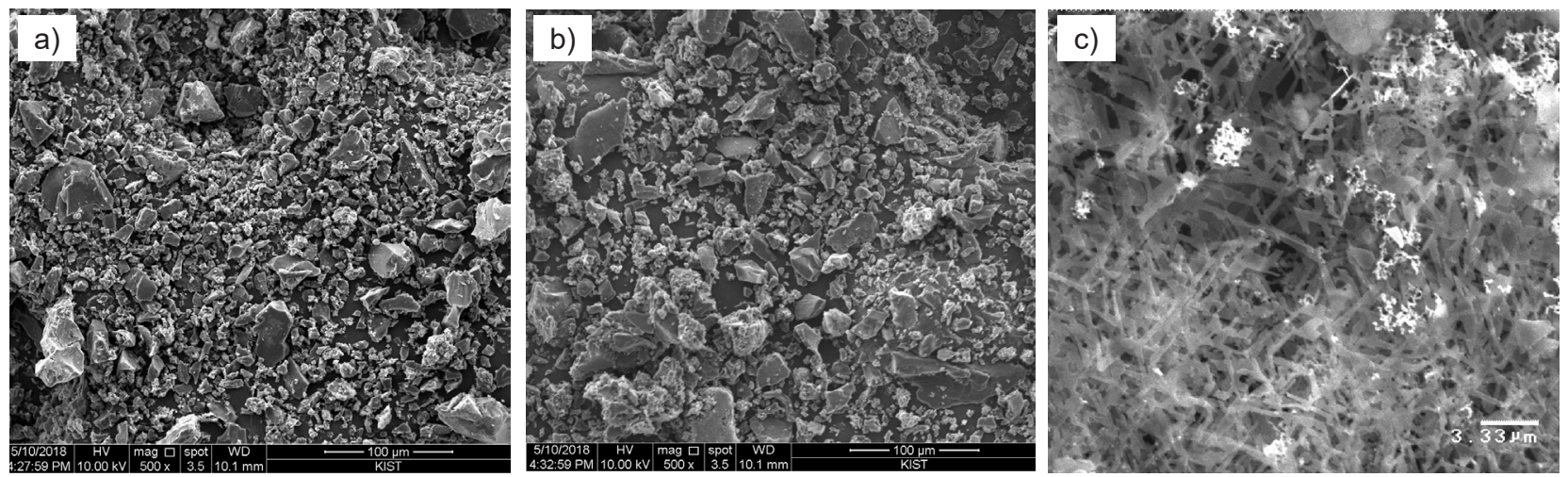

Fig. 3. SEM images of a) primary activated carbon, b) AC treated under supercritical water condition, and c) AC-Ag nanocomposite.

of the carbon atoms. It corresponds to the valence deformation of the $\mathrm{sp}^{2}$ carbon atom on the aromatic hexagonal plane. The appearance of a "D-band" at

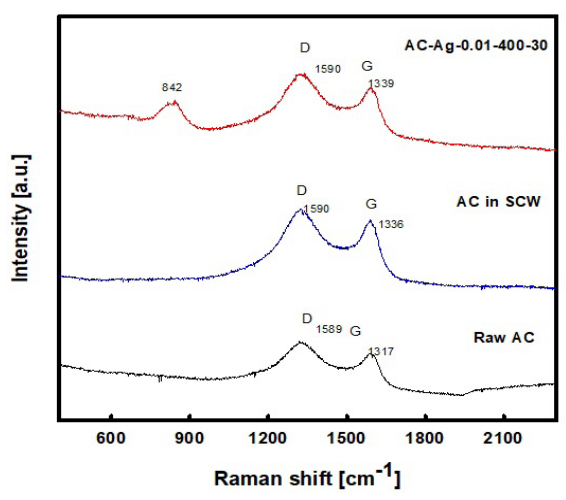

Fig. 4. Raman spectra of the primary activated carbon, activated carbon treated under SCW, AC-Ag nanocomposites prepared under supercritical condition

the shift at $1317-1339 \mathrm{~cm}^{-1}$ refers to the shift mode of the secondary resonance, which mainly reflects the defects in the crystal lattice structure and the in-ordered graphitic structure of the carbon atoms.

The Raman shift at $1590 \mathrm{~cm}^{-1}$ represents the C-C valence deformation, and the peak at the shift of the $1335-1339 \mathrm{~cm}^{-1}$ is related to the active deformation of the hydroxyl group $-\mathrm{OH}$, the carbonyl group $-\mathrm{C}=\mathrm{O}$, the carboxyl group $-\mathrm{COOH}$, and the amino $\mathrm{NH}$ group (Table 3). According to the literature review, depending

Table 3. Raman spectrum interpretation

\begin{tabular}{lccc}
\hline \multirow{2}{*}{ Functional groups } & \multicolumn{3}{c|}{ Raman shift $\left(\mathrm{cm}^{-1}\right)$} \\
\cline { 2 - 4 } & $\begin{array}{c}\text { Primary } \\
\text { AC }\end{array}$ & $\begin{array}{l}\text { AC in } \\
\text { SCW }\end{array}$ & AC-Ag \\
\hline $\begin{array}{l}\text { C-C valence } \\
\text { deformation }\end{array}$ & 1589.2 & 1590.5 & 1590.0 \\
$\begin{array}{l}\text {-OH, -C=O, - COOH, } \\
-\mathrm{NH} \text { deformation }\end{array}$ & 1317.5 & 1336.7 & 1339.1 \\
$-\mathrm{C}-\mathrm{O}-\mathrm{O}$ deformation & - & - & 842.0 \\
\hline
\end{tabular}

on the precursor solution and the synthesis methods, the silver particle's vibrational mode presents between $50 \mathrm{~cm}^{-1}$ and $300 \mathrm{~cm}^{-1}[18,19]$. But in our case, the Raman measurements were carried out between the shift of $500 \mathrm{~cm}^{-1}$ and $2300 \mathrm{~cm}^{-1}$. Therefore, it was not possible to find a clear position band for silver particles. However, for the synthesized silver particles on the activated carbon surface (AC-Ag), the peak of the C-O-C deformation at the $842 \mathrm{~cm}^{-1}$ of the Raman shift is noticed due to the interaction of silver atoms with carboxyl groups of $\mathrm{AC}$ surface and resulting $\mathrm{H}$ - ions reduction.

\section{ACKNOWLEDGMENTS}

This research was supported by a fundamental research project from the Ministry of Education, Culture, and Science of Mongolia funded by the Science and Technological Foundation under contract No. ShuSs. $2017 / 27$. The Raman spectra analysis was conducted by Mr. Badmaarag Altangerel at the Nuclear Research Institute in Dubna, Russia.

\section{CONCLUSIONS}

This work has compared the optimal synthesizing conditions of AgNPs and AC-Ag nanocomposites using the supercritical hydrothermal synthesis method. Under supercritical hydrothermal conditions, it is possible to synthesize relatively uniform distributed silver nanoparticles with an average particle size of 30-80 $\mathrm{nm}$. The smaller silver particles on the surface of activated carbon formed faster than the synthesis of plain particles incorporated into the functional group of activated carbon. XRD analysis of both the plain AgNPs and AC-Ag nanocomposite samples indicates that the peak of silver nanoparticles had a $3 \mathrm{C}$ or side-centred cubic structure. It also confirms that the changes in the deformation of the C-O-C bonding illustrated in the Raman spectra may have been associated with those functional groups of activated carbon that interacted with the silver atoms.

\section{REFERENCES}

1. Yoshimura M., Byrappa K. (2008) Hydrothermal 
processing of materials: past, present and future. Journal of Materials Science., 43, 2085-2103. http://doi.org/10.1007/s10853-007-1853-x

2. Yoko A., Aida T., Aoki N., Hojo D., Koshimizu M., et al. (2018) Supercritical hydrothermal synthesis of nanoparticles. Nanoparticles technology handbook., 3, 683-689. http://doi.org/10.1016/ B978-0-444-64110-6.00060-3

3. Arita T., Hitaka H., Minami K., Naka T., Adschiri, T. (2011) Synthesis of iron nanoparticle: Challenge to determine the limit of hydrogen reduction in supercritical water, J. Supercrit. Fluids 57, 183189. http://doi.org/10.1016/j.supflu.2011.01.008

4. Ruiz-Jorge F., Portela J.R., Sánchez-Oneto J., Martínez de la Ossa E.J. (2020) Synthesis of microand nanoparticles in sub- and supercritical water: From the laboratory to larger scales. Appl. Sci., 10, 5508. http://doi.org/10.3390/app10165508

5. Byrappa K., Adschiri T. (2007) Hydrothermal technology for nanotechnology. Progress in Crystal Growth and Characterization of Materials, 53, 117-166. http://doi.org/10.1016/j. pcrysgrow.2007.04.001

6. Dunne P.W., Munn A.S., Starkey C.L., Huddle T.A., Lester E.H. (2015) Continuous-flow hydrothermal synthesis for the production of inorganic nanomaterials. Phil. Trans. R. Soc., A373, 20150015. http://doi.org/10.1098/rsta.2015.0015

7. Li Y., Gan W., Zhou J., Lu Z., Yang Ch., Ge T. (2015) Hydrothermal synthesis of silver nanoparticles in Arabic gum aqueous solutions. Trans. Nonferrous Met. Soc. Chin., 25(6), 2081-2086. http://doi. org/10.1016/S1003-6326(15)63818-3

8. Hayashi H., Yukiya Hakuta. (2010) Hydrothermal synthesis of metal oxide nanoparticles in supercritical water. Materials, 3(7), 3794-3817. http://doi.org/10.3390/ma3073794

9. Fang Z. (2010) Rapid production of micro- and nano-particles using supercritical water. $1^{\text {st }}$ Edition, Buch. XXVIII, 92 S. Hardcover, ISBN 978-3-64212986-5.

10. Xu Ch., Amyn S., Teja. (2006) Supercritical water synthesis and deposition of iron oxide $\left(\alpha-\mathrm{Fe}_{2} \mathrm{O}_{3}\right)$ nanoparticles in activated carbon. $\mathrm{J}$. of Supercritical Fluids, 39, 135-141. http://doi. org/10.1016/j.supflu.2006.02.004
11. Tayyebi A., Outokesh M. (2016) Supercritical synthesis of a magnetite-reduced graphene oxide hybrid with enhanced adsorption properties toward cobalt \& strontium ions. RSC Adv., 6, 1389813913. http://doi.org/10.1039/c5ra19057f

12. Bagheri S., Julkapli M.N. (2016) Effect of hybridization on the value-added activated carbon materials. Int. J. Ind. Chem., 7, 249-264. http://doi. org/10.1007/s40090-016-0089-5

13. Pandey P., Karki B., Lekhak B., Koirala A.R., Sharma R.K., Pant H.R. (2018) Comparative antibacterial study of silver nanoparticles doped activated carbon prepared by different methods. Journal of the Institute of Engineering, 15(1), 187 194. http://doi.org/10.3126/jie.v15i1.27729

14. Lee H.C., Byamba-Ochir N., Shim W.G., Balathanigaimani M.S., Moon H. (2015) Highperformance supercapacitors based on activated anthracite with controlled porosity. J. Power Sources, 275, 668-674. http://doi.org/10.1016/j. jpowsour.2014.11.072

15. Surenjav E., Buyankhishig B., Byamba-Ochir N., Davaadorj N., Song Z., Tegus O. (2021) Synthesis of silver nanoparticles by hydrothermal processing. Solid State Phenomena 323, 1-7. http://doi. org/10.4028/www.scientific.net/SSP.323.1

16. Zhang Z., Zhou F., Lavernia E.J. (2003) On the analysis of grain size in bulk nanocrystalline materials via x-ray diffraction. Metall. Mater. Trans., 34A 1349-1355. http://doi.org/10.1007/ s11661-003-0246-2

17. Kumar D., Bhardwaj K., Singh K., Verma V., Bhatti H.S. Microwave-assisted synthesis and characterization of silver nanowires by polyol process. Appl. Nanosci., 5(7), 881-890. http://doi. org/10.1007/s13204-014-0386-2

18. Ramirez D., Jaramillo F. (2016) Facile one-pot synthesis of uniform silver nanoparticles and growth mechanism. DYNA 83(198), 165-170. http://doi.org/10.15446/dyna.v83n198.48707

19. Mukherjee P., Roy M., Mandal B.P., Dey G.K., Mukherjee P.K., et al. (2008) Green synthesis of highly stabilized nanocrystalline silver particles by a non-pathogenic and agriculturally important fungus T.asperellum, Nanotechnology 19(7), 075103. http://doi.org/10.1088/0957-4484/19/7/075103 